\title{
Im Schatten der Selbstverständlichkeit - Hürden der Erreichbarkeit unterrepräsentierter Zielgruppen und Grenzen der kompensatorischen Wirkung einer zielgruppengerechten Ansprache
}

\author{
Dr. Stefan Dietrich \\ Fair-Ortung \\ Thomas Maier \\ Fair-Ortung | fair-ortung@posteo.de
}

\section{Zusammenfassung}

Auch internationale Freiwilligendienste sind weit davon entfernt, inklusiv zu sein. Hürden für unterschiedliche Menschen im Zugang zu einem entwicklungspolitischen Freiwilligendienst lassen sich in solche, die im Einflussbereich der Ansprache-Praxis liegen, und solche, die in einem größeren gesellschaftlichen Zusammenhang stehen, unterteilen. Während erstere unmittelbar identifiziert und abgebaut werden können, erfordern letztere zunächst ein tieferes Verständnis gesellschaftlicher Ausgrenzung. Im Rahmen des entwicklungspolitischen Freiwilligendienstes Weltwärts wurden zuletzt vielfältige Anstrengungen unternommen, um die unterrepräsentierten Gruppen „Menschen mit einer Behinderung“, „Menschen in/mit einer Berufsausbildung“ und „Menschen mit einem Migrationshintergrund“ besser zu erreichen und zu begleiten. Basierend auf Erfahrungen von Betroffenen und von Expert*innen, die an Maßnahmen zur Erreichung unterschiedlicher Zielgruppen mitgewirkt haben, wird ein Zugang zu bestehenden Ausgrenzungen herausgearbeitet: Neben dem (Nicht-)Vorhandensein von Rollenmodellen und der (ausbaufähigen) Ausprägung von Selbstvertrauen bei den (und in die!) Zielgruppen deuten sich auch das Verhalten der Programmakteure bestimmende Ausgrenzungsmechanismen an.

Schlagwörter: internationale Freiwilligendienste; Inklusion; Zugangsbarrieren; Ausgrenzung

\begin{abstract}
International voluntary services suffer from a lack of inclusiveness. Generally, people from diverse backgrounds who are interested in realizing a development volunteer service are confronted with two kinds of obstacles. On the one hand there are those which can be confronted through personalized ways of addressing individuals and groups from such backgrounds, and on the other hand more fundamental forms of exclusion. Whereas the former can be identified and tackled readily, the latter require a deep understanding of processes of social exclusion. In recentyears, the development volunteer service Weltwärts has been the sight of numerous attempts to improve the reach and support of underrepresented groups, such as people with disabilities, with a professional formation and with a migratory background. Based on the experiences of affected individuals and experts who are involved in programmes to better reach different target groups, this contribution offers an entry point to existing forms of exclusion. Our focus includes the issue of (inexistent) role
\end{abstract}


models and manifestations of self-confidence (or the lack thereof), but also points to problematic forms of practices from part of the parties involved in providing such services.

\section{Einleitung: Ansprache, Erreichbarkeit und Diversität in entwicklungspolitischen Freiwilligendiensten}

Internationalen Freiwilligendiensten stellt sich die Herausforderung, in einem komplexen, sich ständig wandelnden Umfeld jedes Jahr aufs Neue ausreichend und geeignete Freiwillige zu erreichen. Ein wachsendes Spektrum an alternativen Wegen ins Ausland, sei es durch Angebote im Rahmen der formalen beruflichen Qualifikation (z. B. Berufspraktikum im Ausland, Auslandssemester) oder durch kommerzielle Angebote (sog. Voluntourismus), führt mehr und mehr zu dem Erfordernis, kleiner werdende Jahrgänge junger Menschen möglichst passgenau entsprechend ihrer unterschiedlichen lebensweltlichen Ausdifferenzierungen anzusprechen.

An anderer Stelle (Dietrich 2015; 2016) wurde aufgezeigt, wie eine - von Kenntnissen der aktuellen Jugendforschung informierte - lebensweltlich differenzierte Perspektive zur Verbesserung der Ansprache-Performance durch Trägerorganisationen internationaler Freiwilligendienste bemüht werden kann. Aus ihr lässt sich einerseits eine zielgerichtete Ansprache von Freiwilligendienst-affinen lebensweltlichen Gruppen ableiten, die bei der allgemeinen Bewältigung zurückgehender Bewerbungszahlen schnellen und quantitativen Erfolg verspricht. Andererseits zeigte die Analyse der Ansprache-Performance einzelner Trägerorganisationen, dass besondere Hürden und Hindernisse für Menschen bestehen, die bestimmte Merkmale tragen und die in internationalen Freiwilligendiensten unterrepräsentiert sind.

Die Zusammensetzung der aktuell erreichten Freiwilligen ist äußerst homogen. Wenngleich dies (noch) keine Schwierigkeit bei der Auslastung der zu besetzenden Einsatzplätze darstellt, steht dies dem politischen Willen entgegen, möglichst inklusive Freiwilligendienste anzubieten, die allen Bevölkerungsschichten offenstehen sollen: junge Menschen aus einkommensschwachen Familien, mit einem sogenannten Migrationshintergrund, mit Berufsausbildung, ohne Abitur oder mit einer Beeinträchtigung/Behinderung sind Gruppen, die zwar in der Regel politisch als Freiwillige gewünscht sind, aber im Rahmen der bestehenden Programme und ihrer Formate kaum erreicht werden. Seit Einführung des Freiwilligendienstes Weltwärts beispielsweise oszillierte der Anteil der Teilnehmenden mit Abitur zwischen 96,1 Prozent (2008) und 87,4 Prozent (2015). ${ }^{1}$

1 Der Anteil der Schulabschlüsse mit allgemeiner Hochschulreife im Jahr 2017 lag dabei bei 34,5 Prozent (Statistisches Bundesamt 2018). Siehe AKLHÜ 2018 zu den genannten Zahlen. 
Der entwicklungspolitische Freiwilligendienst Weltwärts - Gemeinschaftswerk des Bundesministeriums für wirtschaftliche Zusammenarbeit und Entwicklung (BMZ) und der zivilgesellschaftlichen Durchführungsorganisationen - bemüht sich vor allem mit eigens dafür eingerichteten Kompetenzzentren um eine Verbesserung der Erreichbarkeit der Zielgruppen Menschen mit einem „Migrationshintergrund“, mit bzw. in einer „Berufsausbildung“ und mit einer „Behinderung/ Beeinträchtigung“. Über diese Kompetenzzentren und die Möglichkeit individueller Begleitmaßnahmen zur Förderung von Inklusion im Weltwärts-Programm (Koordinierungsstelle weltwärts 2016) wurden in den vergangenen Jahren vielfältige Erfahrungen bei der Ansprache, aber auch bei der Begleitung unterschiedlicher unterrepräsentierter Zielgruppen gesammelt. Diese Erfahrungen stellen zum einen ein breites Repertoire an Methoden bereit, mit denen Hürden abgebaut werden können, die im Einflussbereich der Ansprache-Praxis liegen. ${ }^{2}$ Zum anderen stoßen die Expert*innen wie auch wir bei unseren Untersuchungen immer wieder auf grundlegendere, gesellschaftlich tief verankerte Hindernisse, die sich dem unmittelbaren Gestaltungsspielraum der Programmakteure zu entziehen scheinen. Gleichwohl dürfte gerade ein besseres Verständnis solcher grundlegenderen Ausgrenzungsmechanismen dazu beitragen, die Bemühungen um Inklusion zielgerichtet zu fördern, den Ressourcenbedarf realistisch einzuschätzen und Ressourcen effektiv einzusetzen sowie zu einem nachhaltigeren Wandel beizutragen.

Anlässlich des zehnjährigen Jubiläums des Weltwärts-Programms haben wir die Erkenntnisse aus der Ansprache-Studie aus dem Jahr 2015 (Dietrich 2015) um zusätzliche explorative Interviews mit Expert*innen erweitert, die in unterschiedlichen Kontexten in der Ansprache unterrepräsentierter Zielgruppen tätig sind, um mithilfe deren Erfahrungen zusätzlich zur Systematik, mit der die gestaltbare Ansprache-Praxis von Trägerorganisationen ausgewertet und zielführend ausgerichtet werden kann, einige Muster von wirkungsmächtigen Ausgrenzungsmechanismen zu identifizieren.

\section{Vorgehensweise}

Zur Herausarbeitung der Systematik zur Analyse der digitalen Ansprache-Performance von Trägerorganisationen internationaler Freiwilligendienste wurde eine Literaturauswertung aus dem Bereich der Jugendforschung (v. a. Shell-Studie 2010; Sinus-Studie 2012), mit Fokus auf ehrenamtliches Engagement und bestehende Motivationen für einen Freiwilligendienst, vorgenommen, sowie die Webpräsenzen von fünf größeren Trägerorganisationen untersucht (Dietrich 2015; 2016).

2 Siehe hierzu beispielsweise zusammenfassend den Praxiskatalog weltwärts auf dem Weg zur Inklusion (Koordinierungsstelle weltwärts 2016). 
Zur Identifikation von Hürden und Hindernissen wurden mit zwei jungen Menschen, die sich unterrepräsentierten Zielgruppen zuordnen lassen, Nutzer*innentests durchgeführt: Nach der Erprobung zweier ausgewählter Webseiten anhand eines Fragenkatalogs wurde je ein Interview über diesen Test geführt sowie über Erfahrungen und Einschätzungen hinsichtlich internationaler Freiwilligendienste im Allgemeinen (Dietrich 2015; 2016). Außerdem wurden (inklusive der Interviews in Dietrich 2015; 2016) insgesamt sieben problemzentrierte Interviews (Witzel 1989) mit zehn Expert*innen geführt, die in den letzten Jahren in unterschiedlichen Kontexten in der Ansprache und Begleitung unterschiedlicher unterrepräsentierter Zielgruppen tätig gewesen sind. Die Auswahl der Expert*innen orientierte sich an der Abdeckung eines möglichst breiten Spektrums der Zielgruppen. Die interviewten Expert*innen und deren Organisationszugehörigkeit werden vertraulich behandelt. Leitthemen der Interviews waren Inhalte der unternommenen Maßnahmen, Erfahrungen und Ergebnisse bei der Umsetzung, Erklärungsansätze für Erfolge und Misserfolge sowie Anregungen für künftige Bemühungen.

Während Abschnitt 3 die herausgearbeitete Systematik zur Analyse der (digitalen) Ansprache-Performance von Trägerorganisationen internationaler Freiwilligendienste in Bezug auf Hürden für unterrepräsentierte Zielgruppen zusammenfasst, werden in den Abschnitten 4 und 5 die grundlegenderen Ausgrenzungsmechanismen diskutiert, die die durchgeführten User-Tests und problemzentrierten Interviews nahelegen. Abschnitt 6 weist schließlich auf, welche Zugänge zu unterrepräsentierten Zielgruppen grundsätzlich erfolgsversprechend sind und mit welchem Ressourcenbedarf dabei zu rechnen ist. Die Darstellung der Ausgrenzungsmechanismen und der Vorschläge für erfolgsversprechende Zugänge ist dabei die pointierte Synthese der fallanalytischen Interpretation der Interviews durch die Autoren. Sie sollen einen Orientierungsrahmen bilden für weiterführende Untersuchungen, in die insbesondere die Perspektiven und Erfahrungen von Betroffenen stärker einbezogen werden sollten.

\section{Im Einflussbereich der Ansprache-Praxis}

Um die Ansprache-Praxis von Trägerorganisationen internationaler Freiwilligendienste zu untersuchen, lassen sich zunächst unterschiedliche Orte der Ansprache unterscheiden:

Die direkte Ansprache liegt im unmittelbaren Einflussbereich der Trägerorganisationen. Sie lässt sich in die Unterkategorien digitale Ansprache (auf Homepages und in sozialen Netzwerken) und analoge Ansprache (Printmedien, Messen, Schulbesuche, Info-Veranstaltungen, Beratungsgespräche) unterteilen.

Die indirekte Ansprache entzieht sich dagegen dem unmittelbaren Einfluss der Träger. Sie verweist auf die hohe Reproduktionskraft des jeweiligen sozialen Umfelds (Familie, 
Freundeskreis, Mitschüler*innen, Nachbarschaft) junger Menschen. So finden zum Beispiel nicht alle Jugendlichen in gleichem Maße Vorbilder im Familien- und Freundeskreis oder in ihren Schulen, die ihnen nicht nur die Möglichkeit, sondern auch die Machbarkeit und damit das Selbstvertrauen vermitteln, dass ein Freiwilligendienst eine sinnvolle und anstrebenswerte Option der Lebensgestaltung sein könnte.

Die unterschiedlichen Orte der Ansprache lassen sich daraufhin hinsichtlich der Differenziertheit der Ansprache, der Pragmatik der Ansprache und möglicher Hürden und Hindernisse untersuchen.

Wie differenziert die Ansprache erfolgt, bezieht sich dabei zum einen sowohl auf die verschiedenen Unterkategorien digitaler und analoger Ansprache, die angewendet werden, als auch auf die Vielfalt der Optionen, die sie ihren Zielgruppen offerieren, zum Beispiel, an welche biographischen Momente (nach der Schule, nach dem Studium, nach der Ausbildung) sie anschlussfähig sind, welche unterschiedlichen Programme angeboten werden, welche unterschiedlichen Einsatzdauern möglich sind und in welchen Ländern und welchen Tätigkeitsbereichen Freiwillige aktiv sein können. Zum anderen geht es bei der Differenziertheit auch darum, inwieweit unterschiedliche Zielgruppen explizit angesprochen werden (z. B. die Benennung einer grundsätzlichen Offenheit für Menschen mit Behinderung oder der Wunsch nach diversen Freiwilligenteams).

Die Pragmatik der Ansprache fokussiert auf das Wie der Ansprache. Dazu gehören der erste Eindruck, den eine bestimmte Unterform der Ansprache hinterlässt (entscheidend für das „dran bleiben“), das Verhältnis von altruistischen und egotaktischen Motiv(ation)en für einen Freiwilligendienst, die Sprache und Anschaulichkeit der Darstellungen sowie die Breite und der Tiefgang der transportierten Informationen.

Wie differenziert und pragmatisch die Ansprache-Praxis ausfällt, ist dabei zum einen abhängig von den zur Verfügung stehenden Ressourcen. Zum anderen gibt es aber auch „Fallstricke“, die - oft nicht intendierte - Hürden aufbauen. Diese können bei der digitalen Ansprache neben einem schlechten ersten Eindruck in der Zugangslogik der Internetseiten, in einer unter Textlast leidenden Darstellung, im angeforderten Format von Bewerbungen oder in den formulierten Anforderungen liegen. Letztere stellen ein gutes Beispiel dafür dar, wie auf bestimmte Zielgruppen ausgrenzend wirkende Mechanismen sich auf der Ebene der digitalen Ansprache entfalten können, wie diesen hier jedoch gleichzeitig auch entgegengewirkt werden kann.

Im Gesamteindruck der fünf analysierten Webpräsenzen sind die formulierten Qualifikationsanforderungen eher niedrig. Der Freiwilligendienst als Lerndienst wird in den Vordergrund gestellt. Wichtiger als harte Qualifikationen sind Haltungen wie zum Beispiel Toleranz, Neugierde und Interesse an interkulturellen Begegnungen oder entwicklungspolitischen Fragestellungen, aber auch Durchhaltevermögen, 
Anpassungsfähigkeit und Flexibilität. Die zentralen und oft einzigen „hard skills“ stellen die für den Einsatz erforderlichen Sprachkenntnisse dar.

Während dies auf den ersten Blick dafürspricht, dass hinsichtlich der mitzubringenden Fähigkeiten und Eigenschaften die Hürden weitestgehend niedrig gehalten sind, legen die Eindrücke aus den Nutzer*innentests nahe, dass hier auch eine alternative Interpretation denkbar ist. Die geforderten Sprachkenntnisse, aber auch die Vorstellung, in einer sozialen Einrichtung mitzuarbeiten und zum Beispiel unterrichten oder Kinder betreuen zu müssen, wurden als große Herausforderung gesehen, verbunden mit der Frage, ob man selbst überhaupt geeignet und ausreichend ausgebildet dafür ist. Wichtig erscheint in diesem Zusammenhang, dass hier nicht (nur) die tatsächlich vorhandenen Kompetenzen eine Rolle spielen, sondern vielmehr (auch) die Selbsteinschätzung dieser Kompetenzen, wobei sich bei den privilegierten Lebenswelten eine höhere Wahrscheinlichkeit der Überschätzung, in den benachteiligten Lebenswelten dagegen der Unterschätzung vermuten lässt.

Auch Haltungen wie Offenheit oder Flexibilität hängen eng mit dem Selbstverständnis der Interessent*innen zusammen. Es ist daher zu hinterfragen, inwieweit die wenigen genannten Anforderungen nicht bestimmten Lebenswelten und deren Selbstbild näherliegen als anderen. So könnte eine Hürde auch darin bestehen, dass andere Anforderungen, die zum Beispiel bildungsferneren Jugendlichen oder Auszubildenden näherliegen würden, gerade nicht genannt werden, über die sie aber ihre grundsätzliche „Eignung“ leichter erkennen könnten. Schon einmal in einem Betrieb gearbeitet zu haben und alleine gewohnt zu haben oder in einem interkulturellen Umfeld aufgewachsen zu sein, könnte ebenso förderlich sein, eine*n geeignete*n Freiwillige* $n$ abzugeben wie eine offene und an allem interessierte Grundhaltung, die man durch den Genuss einer privilegierten Schulbildung erworben hat.

Während sich viele Hindernisse innerhalb der digitalen Ansprache (aber auch in der analogen Ansprache) prinzipiell identifizieren und abbauen oder zumindest abschwächen lassen, so stößt selbst eine optimierte Ansprache-Performance an Grenzen: Zum einen hilft diese nicht weiter, wenn Angehörige unterrepräsentierter Zielgruppen gar nicht erst auf die Seite mit der zielgruppendifferenzierten Ansprache und dem niederschwelligen Bewerbungsverfahren gelangen; nicht nur, wenn sie von der Existenz der Programme nicht wissen, sondern auch dann, wenn sie sich nicht als berechtigte Adressat*innen erkennen oder sich als grundsätzlich nicht geeignet einschätzen.

Zum anderen bestehen für bestimmte Zielgruppen grundlegende Hürden auch in der Struktur und in der Durchführung der Freiwilligendienste (z. B. auf Abiturjahrgänge optimierte Ausreisezyklen, in der Dauer zu unflexible Programmformate oder keine Fördermöglichkeit zusätzlicher Bedarfe), die nicht alleine durch eine veränderte Ansprache behoben werden können. 


\section{Ausbaufähiges Selbstvertrauen und fehlende Rollenmodelle}

Neben vielen praktischen Hürden, die sich konkret benennen lassen und die zum Teil in der Art der Ansprache oder in der Struktur und Umsetzung der Programme liegen, deuten sich in der Auseinandersetzung mit Nutzer*innen sowie mit den interviewten Expert*innen immer wieder auch grundlegendere Ausgrenzungsmechanismen an, denen eine nicht zu unterschätzende Wirkung auf die Handlungsweisen und den Handlungsspielraum der Beteiligten innewohnen dürfte. Dies sind auf der einen Seite Ausgrenzungsmechanismen, die Menschen, die Merkmale unterrepräsentierter Zielgruppen tragen, daran hindern, einen Zugang zu den prinzipiell offenen Dienstangeboten zu finden. Auf der anderen Seite finden sich Ausgrenzungsmechanismen, die die Freiwilligendienste anbietenden Organisationen daran hindern, offen auf die unterrepräsentierten Zielgruppen zuzugehen bzw. mit ihnen umzugehen.

Beim Selbst-Ausgrenzen der Betroffenen stellen ein ausbaufähiges Selbstvertrauen und fehlende Rollenmodelle zentrale Mechanismen dar. Die in Abschnitt 3 skizzierte Selbsteinschätzung in den Nutzer*innentests bezüglich der geforderten Fähigkeiten ist ein Beispiel für ein schicht- und lebensweltspezifisch unterschiedlich ausgeprägtes Selbstvertrauen. Entscheidend hierbei ist die Annahme, dass es sich nicht nur um persönlichkeitsbedingte Unterschiede in der Ausprägung des Selbstvertrauens handelt, sondern dass ein ausbaufähiges Selbstvertrauen bei Träger*innen bestimmter Merkmale (auch) die Verinnerlichung wiederholter Diskriminierungserfahrungen aufgrund dieser Merkmale im Rahmen ihrer Sozialisation darstellt. Eine solche Verinnerlichung von gesellschaftlich vermittelten Diskriminierungen kann als eine zu kompensierende Benachteiligung kategorisiert werden, die einen proaktiven Umgang seitens der an Inklusion interessierter Beteiligter erfordert.

Zur Reproduktion dieser Ausgrenzung trägt entsprechend das Fehlen von Erfahrungen im Feld der internationalen Freiwilligendienste seitens des direkten Umfeldes oder von anderen Merkmalsträger*innen der unterrepräsentierten Gruppen bei. Der Austausch über gemachte Erfahrungen kann eine entscheidende Hilfe bei der Entscheidungsfindung darstellen, da an dieser Stelle Hoffnungen, Erwartungen und Ängste kommuniziert und abgearbeitet werden können. In Lebenswelten, in denen solche Formen des Engagements nicht oder selten vorkommen, in denen unter Umständen gar eine große Skepsis gegenüber solchen Programmen herrscht, werden einer positiven Evaluierung der Machbarkeit eines Dienstes deutliche Hürden in den Weg gelegt.

\section{Berührungsängste und Projektionen}

Auch bei den Programmakteuren internationaler Freiwilligendienste (und bei externen Akteuren) sind Mechanismen zu erkennen, die gegenüber unterrepräsentierten Zielgruppen ausgrenzend wirken. Neben einer bestätigenden Spiegelung des 
mangelnden Selbstvertrauens (z. B. durch Verantwortliche von Einrichtungen für Menschen mit Behinderung, die ihren Klient*innen einen entwicklungspolitischen Freiwilligendienst nicht zutrauen) sind bei diesem Andere-Ausgrenzen Deutungsmuster auffällig, die auf Berührungsängste gegenüber bestimmten Zielgruppen hindeuten sowie auf Projektionen der eigenen Unsicherheiten auf andere Beteiligte oder „die Gesellschaft“. So können Argumentationen wie beispielsweise, „noch nicht soweit zu sein“, „nicht über ausreichende Ressourcen zu verfügen“, „die Partnerorganisationen nicht überfordern zu wollen“ etc. nicht ausschließlich auf strukturellen Hürden (z. B. keine personellen Ressourcen angesichts komplexer werdender Anforderungen), auf Nichtwissen (z. B. um Modelle der Finanzierung oder vielversprechende Wege der Implementierung) oder die mangelnde Bereitschaft anderer beruhen. Vielmehr kann diesen auch eine abwehrende bzw. ablehnende Haltung gegenüber der im Allgemeinen bejahten Forderung nach mehr Inklusion in internationalen Freiwilligendiensten zugrunde liegen. Effektive Schritte zur Inklusion werden über diese Argumentation häufig vertagt oder sehr zögerlich angegangen. Problematisch erweist sich hierbei auch die generell ungenügende Evaluation, Dokumentation, Aufbereitung und Streuung der Erfahrungen mit der Arbeit mit unterrepräsentierten Zielgruppen, trotz der Anstrengungen der Kompetenzzentren auf diesem Feld. Berührungsängste und Unsicherheiten setzen sich zudem bei der Umsetzung im Rahmen der Begleitung einmal gewonnener Freiwilliger aus unterrepräsentierten Zielgruppen fort. Hierbei stellt sich auch die Frage nach der Homogenität bzw. der Unterrepräsentation bestimmter Gruppen bei den pädagogisch Begleitenden. Sowohl die Auswahlmechanismen, als auch die Erwartungshaltungen gegenüber potentiellen bzw. tatsächlichen Teilnehmenden können nicht frei von Machtstrukturen und gelernten, eingeübten und oft unhinterfragten Prämissen funktionieren. Dies erfordert eine Reflexion, inwiefern eigene biographische Prägungen, Interessen und Lebenswelten die Auswahlmechanismen sowie die Qualität der Begleitung beeinflussen. Dabei erscheint es uns als unerlässlich, dass alle Beteiligten sich kritisch mit den Effekten von gelernten, aber nicht präsenten Vorurteilen und Stereotypen auseinandersetzen (Greenwald/Krieger 2006).

\section{Zugänge und Aufwände}

Die Erfahrungen von Maßnahmen zur Erreichung unterrepräsentierter Zielgruppen zeigen, dass es viel an individueller Aufbauarbeit und vermittelnden Personen/ Institutionen bedarf, um die Lücken zwischen den sich und andere ausgrenzenden Lebenswelten (internationale Freiwilligendienste auf der einen, unterrepräsentierte Gruppen auf der anderen Seite) zu überbrücken. ${ }^{3}$

So ist es erstens von grundsätzlicher Bedeutung, Wege der Sensibilisierung zu finden, um bestimmte Problemzusammenhänge für alle am Prozess Beteiligten überhaupt

3 Siehe exemplarisch hierzu In Via Köln e. V. (2016). 
sichtbar und sagbar und damit einer Anerkennung zuführbar zu machen, da diese sich oft hinter individualisierten Zuschreibungen und milieuspezifisch geprägten Wahrnehmungen verstecken und sich damit im Schatten der jeweils eigenen lebensweltlichen Selbstverständlichkeit bewegen. Dies gilt für konkrete praktische Hürden genauso wie für die skizzierten grundlegenderen Ausgrenzungsmechanismen.

Zweitens ist für die Ansprache eine eigene Sprache zu erlernen, die den Zugang unterrepräsentierter Gruppen zu den Programmen erleichtert, zum Beispiel durch Peer-to-Peer-Ansätze (Peers als Multiplikator*innen), aber auch durch die Nutzung der Expertise und Erfahrungen von Interessenvertretungen, die einen direkten Zugang zu einer der Zielgruppen haben bzw. diese repräsentieren.

Drittens sind in der Umsetzung dezentrale, lokal verankerte Ansätze erforderlich, insofern, dass es von zentraler Bedeutung sein wird, junge Menschen aus entsprechenden Zielgruppen an ,ihren“ Orten direkt aufzusuchen. Das können zum Beispiel Schulen, Sportvereine, Jugendzentren, aber auch Ausbildungsbetriebe sein, die unter anderem auch über Personen verfügen, die vonseiten der jungen Menschen über großes Vertrauen verfügen und als Scharniere der Motivation, aber im weiteren Verlauf auch der Vorbereitung dienen können.

Dies heißt viertens, dass diese Ansprachen nur als langfristige Heranführung und Begleitung erfolgversprechend wirken können. Dies umfasst nicht nur die Arbeit mit den jungen Menschen selbst, sondern auch das Bemühen, das Umfeld der Interessierten (z. B. Elternberatungen, Vereinbarungen mit Ausbildungsbetrieben, Kooperation mit Einrichtungen für Menschen mit Behinderung) für einen internationalen Freiwilligendienst zu begeistern und so Sorgen und Bedenken abbauen und Perspektiven entwickeln zu helfen.

Für die ersten beiden Punkte ist ein besonderer Zugang erforderlich, der sich vermutlich nicht ohne Weiteres aus den bestehenden Wissens- und personellen Erfahrungsbeständen der Trägerlandschaft internationaler Freiwilligendienste erschließen lässt. So wäre es an dieser Stelle von strategischer Bedeutung, mehr verstehende Wissenszugänge zu den ausgegrenzten Gruppen zu fördern und auf eine Gewinnung von Multiplikator*innen mit Zugangskompetenz bzw. die Inklusion solcher Kompetenz in die eigenen Reihen hinzuwirken. Die hier herausgearbeiteten Überlegungen zu Zugangsbarrieren in internationalen Freiwilligendiensten basieren zu einem großen Teil auf den Erfahrungen von Expert*innen, die im Bereich der Inklusion von unterrepräsentierten Zielgruppen tätig sind. Für künftige Untersuchungen wären qualitative, ethnographische Zugänge sowohl zu den Erfahrungen einzelner Freiwilliger (retrospektiv, aber vor allem auch begleitend), als auch zu den oben genannten sozialen Orten der jungen Menschen aus unterrepräsentierten Zielgruppen vielversprechend. Darüber ließen sich Ausgrenzungsmechanismen und Bewältigungsstrategien in unterschiedlichen Kontexten besser fassen und verstehen sowie 
von wissenschaftlicher Seite ein Beitrag zum Erlernen einer für die Zielgruppenansprache eigenen Sprache leisten, der sensibel ist für die oft unscheinbaren, aber wirkungsmächtigen Sequenzen lebensweltlicher Aus- und Abgrenzung.

Für die zweiten beiden Punkte sind der hohe Aufwand und der beträchtliche Zeithorizont zu bedenken, der angesichts der notwendigen Dezentralität in der Umsetzung sowie der Vielfalt und Anzahl der spezifischen „Zugangsorte“ anfällt. Hierfür macht die Vielfalt und geographische Streuung der Trägerlandschaft internationaler Freiwilligendienste Hoffnung: wenn es gelingt, die unter den ersten beiden Punkten wachsende Expertise und die erforderlichen Ressourcen den einzelnen Organisationen zugänglich zu machen sowie die vorhandenen Berührungsängste weiter abzubauen. So können die Trägerorganisationen vielschichtig und vielerorts, eben dezentral und lokal verankert auf unterschiedliche Gruppen zugehen womit die Trägerorganisationen einen Beitrag zum gesamtgesellschaftlichen Empowerment der verschiedenen Zielgruppen leisten können.

\section{Literaturverzeichnis}

AKLHÜ (2018): Freiwillige in internationalen Freiwilligendiensten 2017. Statistische Übersicht, Arbeitskreis "Lernen und Helfen in Übersee“ e. V., Bonn, https://www.entwicklungsdienst.de/fileadmin/AKLHUE_Relaunch/Statistische_Erhebung_Outgoing_2017.pdf (31.03.2019).

Dietrich, Stefan (2015): Motivation, Ansprache, Erreichbarkeit. Herausforderungen für Trägerorganisationen internationaler Freiwilligendienste im Zugang zu jungen Menschen, Diskussionspapier Arbeitskreis „Lernen und Helfen in Übersee“ e. V., Bonn, https://www. entwicklungsdienst.de/fileadmin/AKLHUE_Relaunch/Studie_Dietrich_Motivation__ Ansprache__Erreichbarkeit.pdf (31.03.2019).

Dietrich, Stefan (2016): Motivation, Ansprache und Erreichbarkeit. Die „pragmatische Generation" als Herausforderung für Trägerorganisationen internationaler Freiwilligendienste, in: Voluntaris - Zeitschrift für Freiwilligendienste, Jg. 4, Heft 2, S. 154-185

Greenwald, Anthony und Linda Krieger (2006): Implicit Bias: Scientific Foundations, in: California Law Review, J.g 94, Heft 4, S. 945-967.

In Via Köln e. V. (2016): Kompetenzzentrum für Menschen mit Berufsausbildung im Freiwilligendienst weltwärts. Eine Handreichung für Entsendeorganisationen, www.invia-koeln. de/downloads/projekte/internationales/kompetenzzentrum/Kompetenzzentrum-Handreichnung.pdf (31.03.2019).

Koordinierungsstelle weltwärts (2016): weltwärts auf dem Weg zur Inklusion. Engagement im Rahmen von Programmbegleit- und Rückkehrmaßnahmen, Bonn, www.weltwaerts. de/de/weltwaerts-auf-dem-weg-zur-inklusion.html (31.03.2019).

Statistisches Bundesamt (2018): Schulen auf einen Blick,www.destatis.de/GPStatistik/servlets/MCRFileNodeServlet/DEHeft_derivate_00035140/Schulen_auf_einen_Blick_2018_Web_ bf.pdf (31.03.2019).

Witzel, Andreas (1989): Das problemzentrierte Interview, in: Qualitative Sozialforschung in der Psychologie. Grundfragen, Verfahrensweisen, Anwendungsfelder, hrsg. von Gerd Jüttemann, 2. Aufl., Heidelberg, S. 227-256. 